\title{
Conf $9405264-y$
}

LA-UR- 94-3937
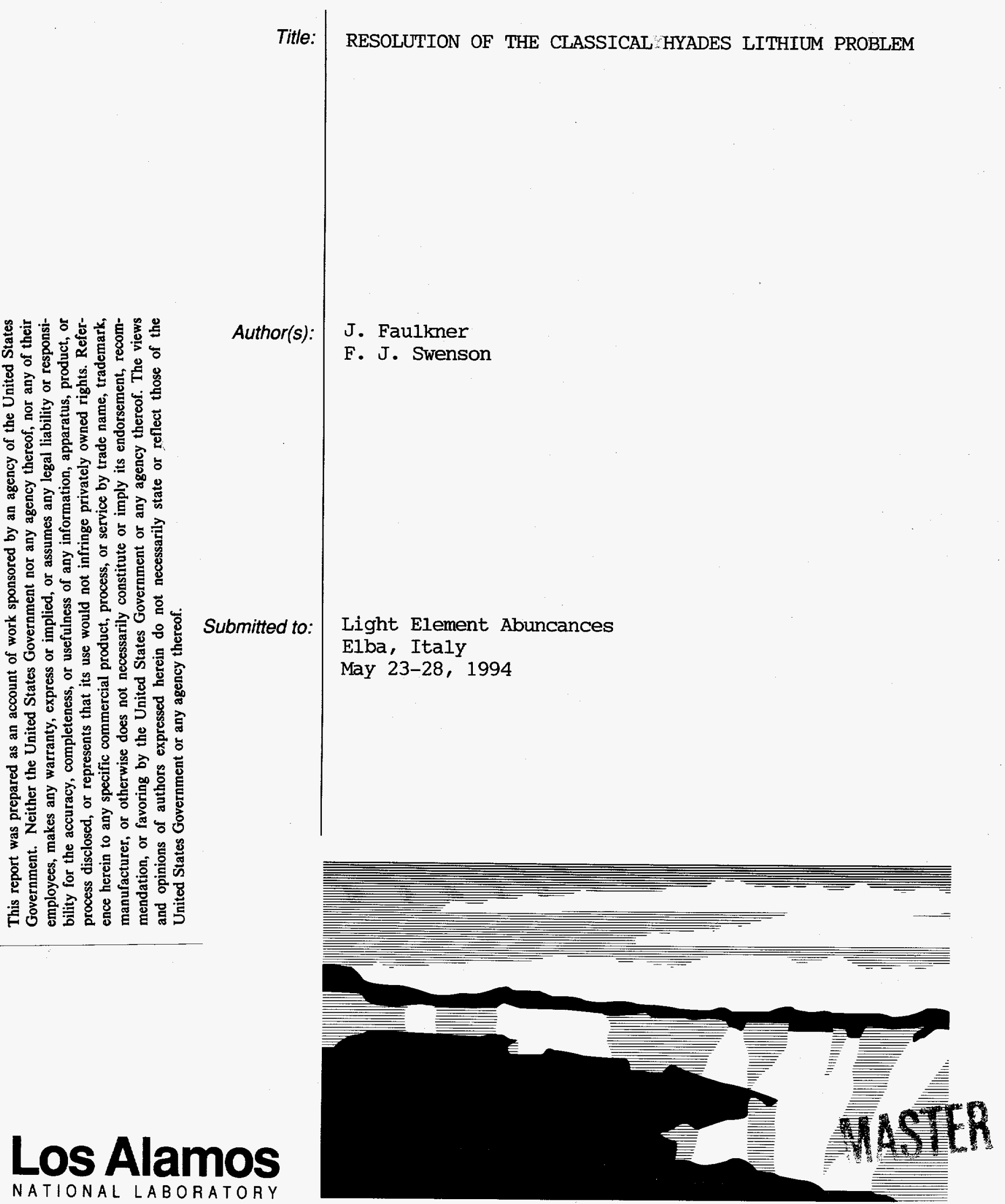

NATIONAL LABORATORY

Los Alamos National Laboratory, an affirmative action/equal opportunity emplo'yer, is operated by the University of California for the U.S. Department of Energy under contract W-7405-ENG-36. By acceptance of this article, the publisher recognizes that the U.S. Government retains a nonexclusive, royalty-free license to publish or reproduce the published form of this contribution; or to allow others to do so, for U.S. Government purposes. The Los Alamos National Laboratory

requests that the publisher identify this article as work performed under the auspices of the U.S. Department of Energy. 6

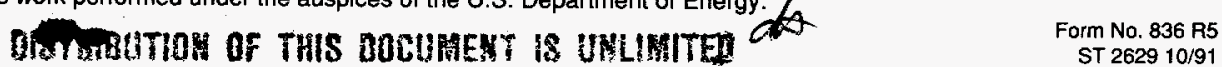




\section{DISCLAIMER}

This report was prepared as an account of work sponsored by an agency of the United States Government. Neither the United States Government nor any agency thereof, nor any of their employees, make any warranty, express or implied, or assumes any legal liability or responsibility for the accuracy, completeness, or usefulness of any information, apparatus, product, or process disclosed, or represents that its use would not infringe privately owned rights. Reference herein to any specific commercial product, process, or service by trade name, trademark, manufacturer, or otherwise does not necessarily constitute or imply its endorsement, recommendation, or favoring by the United States Government or any agency thereof. The views and opinions of authors expressed herein do not necessarily state or reflect those of the United States Government or any agency thereof. 


\section{DISCLAIMER}

Portions of this document may be illegible in electronic image products. Images are produced from the best available original document. 


\title{
RESOLUTION OF THE CLASSICAL HYADES LITHIUM PROBLEM
}

\author{
John Faulkner ${ }^{1}$, Fritz Swenson ${ }^{2}$ \\ ${ }^{1}$ Lick Observatory and Astronomy Board, UC Santa Cruz, \\ Santa Cruz, CA 95064 \\ ${ }^{2}$ Los Alamos National Laboratory, X-2 MS B220, \\ Los Alamos, NM 87545
}

\begin{abstract}
For the very first time, it has recently proved possible to make a wellmotivated, physically plausible, and self-consistent prediction of the Hyades G- and $\mathrm{K}$ $\mathrm{d}$ warf $\left(\mathrm{Li}, \mathrm{T}_{\text {eff }}\right)$ relationship that matches the long-unexplained observations. The method employs the latest Iglesias \& Rogers (OPAL) interior opacities and Alexander surface opacities (whose respective values are now themselves close to empirical predictions or estimates made earlier in this Hyades project), King's recently discovered [O/Fe] enhancement (another prediction!) and utterly conventional PMS (pre-main-sequence) evolution unaided by arbitrary and ad hoc adjustable parameters.
\end{abstract}

Thus, the following assumptions form a self-consistent set explaining the Hyades G- and $\mathrm{K}$-dwarf $\left(\mathrm{Li}, \mathrm{T}_{\text {eff }}\right)$ observations:

(i) Pop. I interior opacities are now essentially correct.

(ii) Pop. I surface opacities are now essentially correct.

(iii) The Hyades distance is now essentially correct.

(iv) The Hyades $[\mathrm{Fe} / \mathrm{H}]$ and $[\mathrm{O} / \mathrm{Fe}]$ are now essentially correct, and possible changes in $[\mathrm{Ne}, \mathrm{Mg}$, or $\mathrm{Si} / \mathrm{Fe}]$ are likely to have only a small effect.

(v) Convective envelope overshooting is negligible.

(vi) Quarreling with the above means finding at least two compensating errors in assumptions (i) through (v).

\section{Introduction}

For some time, we and colleagues have pursued possible resolutions of a now 30year old puzzle - the classical Hyades lithium problem. We have recently reached a most satisfying conclusion, a well-motivated, physically plausible and parameter free self-consistent prediction of the Hyades $\mathrm{G}$ - and $\mathrm{K}$-dwarf $\left(\mathrm{Li}, \mathrm{T}_{\text {eff }}\right.$ ) relationship that matches the long-unexplained observations. Space precluding full discussion, we here present a guide to the main points in our papers. In logical (not published) order, relevant references are SF (Swenson \& Faulkner 1992), SSF 
(Swenson, Stringfellow, \& Faulkner 1990a), SFRI90 (Swenson et al 1990b), SFRI94 (Swenson et al 1994a), SFIRA (Swenson et al 1994b).

\section{The Problem; Earlier Ideas}

Wallerstein, Herbig, \& Conti (1965) noted that [Li/Ca] dropped quite sharply in Hyades $\mathrm{G}$ - and $\mathrm{K}$-dwarfs, correctly suspecting a reduction in $\mathrm{Li}$ with decreasing $\mathrm{T}_{\text {eff }}$. Since standard main-sequence (MS) convective envelopes were too shallow to explain Li-depletion via MS-burning, other explanations were sought. Bodenheimer (1965) seemed to show that conventional pre-main-sequence (PMS) burning was also (marginally?) inadequate; subsequent readers ignored his optimistic summary.

As time elapsed, the problem only seemed to worsen. Successively improved observations showed even stronger Li-depletion (Cayrel et al 1984; see Fig. 1); while reductions in measured $[\mathrm{Fe} / \mathrm{H}]_{\mathrm{Hyad}}$ implied even less theoretical PMS depletion. The widening gap led to an era of $(a d h o c)^{2}$ theories. Ad hoc parameters (e.g., a multiple of the deep envelope scale-height) were introduced to characterize nonstandard features of convection (MS "overshoot" - Straus et al 1976; PMS "extramixing" - D'Antona \& Mazzitelli 1984). When fixed parameter values produced $\left(\mathrm{Li}, \mathrm{T}_{\text {eff }}\right)$ curves markedly at odds with those observed, appeals were made to $a d$ hoc variations in the ad hoc parameters.

\section{Our Own Approach: Deduction, Production, and Use of Improved Opacities}

We first briefly discuss how we eliminated another earlier promising idea.

\subsection{Main-Sequence Mass-Loss Doesn't Work}

Very early on, Weymann \& Sears (1965) briefly explored a suggestion by N.J. Woolf, that mass-loss might lead to envelope Li-depletion (currently Li-rich material being lost while previously hotter, Li-free material continuously dilutes the envelope). They sketched the $\mathrm{Li}$ consequences - an ultimately exponentially decaying dependence on mass lost - of assuming an unchanging envelope mass for a given model. Hobbs et al (1989) did much the same.

However, the key to ruling out mass-loss lies in the realization that MS convective envelope masses vary (almost linearly!) with stellar mass. This happy circumstance yields quasi-analytic predictions for Li-depletion that not only match properly computed results very closely (SF), but also run along or parallel to most of the $\mathrm{G}$ - and $\mathrm{K}$-dwarf $\left(\mathrm{Li}, \mathrm{T}_{\text {eff }}\right)$ observations. Explaining the observations then requires that those dwarfs all started out with very closely defined masses (e.g. $1.08 \pm 0.01 M_{\odot}$ ), before losing various amounts of mass; a circumstance so contrived that it rules out this kind of explanation. 
Fig. 1. The Hyades (Li, $\mathrm{T}_{\text {eff }}$ ) plane. Observations (crosses, open squares, and circles) are from Cayrel et al (1984), Duncan \& Jones (1983), and Boesgaard \& Trippico (1986), respectively. Thorburn et al (1993) have recently published more observations that support and supplement the Cayrel et al data (see Fig. 2 of SFIRA). Arrows (one, at $\log N(\mathrm{Li}) \sim 0.5$, obscured) indicate upper limits. Computed results are normalized to initial $\log N\left({ }^{7} \mathrm{Li}_{\mathrm{o}}\right)=3.2$ (long dashed line) on a scale where $\log N(\mathrm{H})=12.0$. Solid symbols give model results. Mass increments between models are $0.05 M_{\odot}\left(M<1.2 M_{\odot}\right)$ and $0.10 M_{\odot}\left(M>1.2 M_{\odot}\right)$. Triangles identify $1 \mathcal{M} \odot$ models. Sequence A shows the $\left(\mathrm{Li}, \mathrm{T}_{\text {eff }}\right)$ predictions for models with older fiducial physics. Sequence SSF is the fit produced with a 37 percent maximum enhancement of Cox \& Tabor (1976) interior opacities as described in SSF.

Fig. 2. Model predictions in the Hyades $\left(\mathrm{Li}, \mathrm{T}_{\text {eff }}\right)$ plane. See Figure 1 for general description. Sequences, computed with different inputs or physics, are labeled A, B, C, D, and $F$ and are described in SFRI94. They show the non-monotonic influence of successive changes on self-consistent predictions. Note that all $1 M_{\odot}$ models have roughly the same temperature, an empirical constraint resulting from the required consistency with the solar constraint and Hyades M-L information.

\subsection{The Importance and Influence of Opacities for PMS Li-Burning}

While working on mass-loss, we became aware not only that extant PMS studies relied on older opacities, but also that a careful systematic examination of the separate influence of interior and surface opacities had never been made. As we were to show (SSF), increments in these separate opacities have oppositely signed effects on PMS envelope evolution and therefore ultimate Li-content. (Simply put, the surface opacity helps determine which adiabat the deep envelope attains, while the interior opacity determines where the radiative interior leaves the adiabat Faulkner \& Swenson, in preparation.)

At the time, upward historical opacity trends were clear, although, for example, Iglesias \& Rogers were yet to turn their attention to opacities for $\mathrm{T}>10^{6} \mathrm{~K}$. (For 
PMS burning, the base of the envelope reaches its maximum temperature for only a few $\times 10^{6}$ yrs as a $\sim 1 M_{\odot}$ star begins to develop a radiative core - and therefore a retreating convective envelope - and thus moves from a Hayashi to a radiative contracting track. This time-scale sets the important temperature for PMS Liburning at $\mathrm{T} \sim 4 \times 10^{6} \mathrm{~K}$, not the $\sim 2.5 \times 10^{6} \mathrm{~K}$ commonly misquoted.)

SSF made an end run around uncertain and gradually changing theoretical opacities by asking what the internal opacities would need to become (temporarily assuming unchanged surface opacities), to match the $\mathrm{Li}$ observations. Figure 1 (SFRI94) essentially summarizes the dramatic consequences SSF found with a procedure involving a $\sim 37 \%$ increment over Cox \& Tabor (1976) opacities. While SSF did not address the full question of consistency with other Hyades data, one consequence of the approach was both startling and gratifying - the observations, "like beads on a string" (Burt Jones), now lay along our theoretical curve. Unlike all other attempted explanations this was the first time that a close match to the characteristic curve was obtained; it remained a robust feature of our results as work continued.

In unpublished work, we allowed for a further $50 \%$ increase in surface opacities, finding that internal increments of $\sim 45-48 \%$ would then fit the data. Spot check opacities for $\mathrm{T} \sim 3.5$ and $4 \times 10^{6} \mathrm{~K}$, kindly computed by Iglesias \& Rogers, indeed matched these latter requirements closely! However, part of the change could be due to revised heavy element mixtures. To learn what change could be attributed solely to a change in opacity-computing methodology, Iglesias \& Rogers undertook to recompute interior opacities for a mixture (King IVa) employed by Cox \& Tabor. The resulting differences were substantial, if not as large as before. In SFRI90 we showed the ( $\left.\mathrm{Li}, \mathrm{T}_{\text {eff }}\right)$ consequences of this change in internal opacity code: a removal of some $75-80 \%$ of the discrepancy. When we permitted ourselves (unpublished) to add a little helium, to restore the original mass-luminosity (M-L) relationship - leaving aside whether it was absolutely correct - a further 10-15\% of the discrepancy was removed. (Sequences analogous to all three here described were later shown in Fig. 4 of SFRI94.)

The stage was now set for a massive undertaking (SFRI94) - to require that the spectroscopic $[\mathrm{Fe} / \mathrm{H}]$ and the $\mathrm{M}-\mathrm{L}$ relationship with the best-determined distance should both be fitted, predicting the ( $\mathrm{Li}, \mathrm{T}_{\text {eff }}$ ) consequences (and, incidentally, the H-R diagram) for a succession of changes in physical inputs: internal opacities, surface opacities, heavy element mixes, equation-of-state, etc. (As with our change in opacity code only - but not mixture - in SFRI90, such careful isolation of effects is not common in the field.) Whenever a physical input was changed, we re-calibrated the mixing-length parameter $\alpha$ by re-evolving a solar model with the same physics, and completed the $[\mathrm{Fe} / \mathrm{H}]$ and $\mathrm{M}-\mathrm{L}$ self-consistent procedure described in SFRI94. Interested readers can there follow the ebb and flow in $\left(\mathrm{Li}, \mathrm{T}_{\text {eff }}\right)$ predictions as five major input improvements are pursued. All but one are shown in Fig. 2.

It is historically ironic that the prediction of an excellent fit to the H-R diagram (Fig. 2b of SFRI94) is an incidental consequence. One of us (Faulkner 1964) first pointed out that the Hyades M-L and H-R data could simply not be fitted simultaneously with the then distance modulus of 3.03 mag; he suggested moving 
the Hyades away! The present excellent $\mathrm{H}-\mathrm{R}$ prediction using a distance modulus of $3.35 \pm 0.02 \mathrm{mag}$ (Peterson \& Solensky 1988) serves as a remarkable confirmation of the general correctness of this much greater value.

Final SFRI94 Li predictions (sequence F; see Fig. 2) were much closer to the observations than sequence $\mathrm{A}$, but still left room for improvement. We showed that four relatively modest input changes could each produce an essentially perfect fit. One such change, noting that oxygen was second only to iron in providing metallic opacities at $\mathrm{T} \sim 4 \times 10^{6} \mathrm{~K}$, was a modest relative oxygen enhancement. Shortly after SFRI94 was submitted, we heard that Jeremy King had quite independently found that the Hyades $[\mathrm{O} / \mathrm{H}]$ was indeed somewhat higher than $[\mathrm{Fe} / \mathrm{H}]$, at $0.265 \pm 0.05$ versus $\sim 0.12$ or $0.13 \pm 0.03$.

Thus, in our final letter, SFIRA, we examined the consequences of a "low" oxygen enhancement, $\mathrm{OL}$, with $[\mathrm{O} / \mathrm{H}] \sim 0.2$, and a "high" enhancement, $\mathrm{OH}$, with $[\mathrm{O} / \mathrm{H}] \sim 0.3$. Special sets of interior opacities (Iglesias \& Rogers) and surface opacities (Alexander) were computed, with oxygen specifically enhanced. Prior to using these, we updated sequence $\mathrm{F}$ with several small improvements to various inputs. Sequence $F$, its update, and sequences $\mathrm{OL}$ and $\mathrm{OH}$ are shown in Figure 3. The $\mathrm{OH}$ prediction (within King's upper limit) essentially fits the observations. As a measure of how good the predictions are, "extra-mixing" by only $\sim 0.04 H_{p}$ would bring the OL results into perfect agreement (compared to D'Antona \& Mazzitilli's $0.7 H_{p}$ for a poor quality shoulder region fit - see Fig. 7 of SFRI94). We also examined the separate influence of up to 18 elements on relevant opacities (Fig. 3 of SFIRA); only accurate abundances of key elements so identified can put our (or anyone's) predictions on their firmest footing.

Swenson has continued improving our evolutionary code's equation of state (to give closer agreement with that used in the opacity calculations). With the latest improvement, the revised OL prediction moves down to the position of the $\mathrm{OH}$ curve in Figure 3; thus, the revised $\mathrm{OH}$ and $\mathrm{OL}$ predictions straddle the very small spread of the data about a mean fitting curve.

To summarize: we feel that we and our colleagues have done the best job of predicting the consequences for the $\left(\mathrm{Li}, \mathrm{T}_{\text {eff }}\right)$ Hyades relationship from standard models employing the best physical inputs we can, consistent with all available and known constraints for this best observed and fundamentally important cluster. When we do so, lo and behold, the prediction matches the observations!

\section{References}

Bodenheimer, P. 1965, ApJ, 158, 419

Boesgaard, A.M., \& Tripicco, M.J. 1986, ApJ, 302, L49

Cayrel, R., Cayrel de Strobel, G., Campbell, B., \& Däppen, W. 1984, ApJ, 283, 205

Cox, A.N., \& Tabor, J.E. 1976, ApJS, 31, 271

D'Antona, F., \& Mazzitelli, I. 1984, A\&A, 138, 431

Duncan, D.K., \& Jones, B.F. 1983, ApJ, 271, 663

Faulkner, J. 1964, The Observatory, 84, 257

Hobbs, L.M., Iben, I., Jr., \& Pilachowski, C. 1989, ApJ, 347, 817 
John Faulkner, Fritz Swenson: RESOLUTION OF HYADES Li PROBLEM

Fig. 3. Figure 1 of paper SFIRA. Computed results normalized to $\log N\left({ }^{7} \mathrm{Li}_{\circ}\right)=3.25$ initially. Dashed curve, replotted sequence F ("best" model from SFRI94); dotted curve, updated sequence F (see text); dash-dot curve, sequence OL (lower of two oxygen enhancements); solid line, sequence $\mathrm{OH}$ (higher oxygen enhancement).

Peterson, D.M., \& Solensky, R. 1988, ApJ, 333, 256

Straus, J.M., Blake, J.B., \& Schramm, D.N. 1976, ApJ, 204, 481

Swenson, F.J. \& Faulkner, J. 1992, ApJ, 395, 654 (SF)

Swenson, F.J., Faulkner, J., Iglesias, C.A., Rogers, F.J., \& Alexander, D.R. 1994b, ApJL, 422, L79 (SFIRA)

Swenson, F.J., Faulkner, J., Rogers, F.J, \& Iglesias, C.A. 1990b, BAAS, 22, 1223 (SFRI90)

Swenson, F.J., Faulkner, J., Rogers, F.J, \& Iglesias, C.A. 1994a, ApJ, 425, 286 (SFRI94)

Swenson, F.J., Stringfellow, G.S., \& Faulkner, J. 1990a, ApJL, 348, 33 (SSF)

Thorburn, J.A., Hobbs, L.M., Deliyannis, C.P., \& Pinsonneault, M.H. 1993, ApJ, 415, 150

Wallerstein, G., Herbig, G.H., \& Conti, P.S. 1965, ApJ, 141, 610

Weymann, R., \& Sears, R.L. 1965, ApJ, 142, 174

This book was processed by the author using the $\mathrm{T}_{\mathrm{E}} \mathrm{X}$ macro package from SpringerVerlag. 
Fig 1.

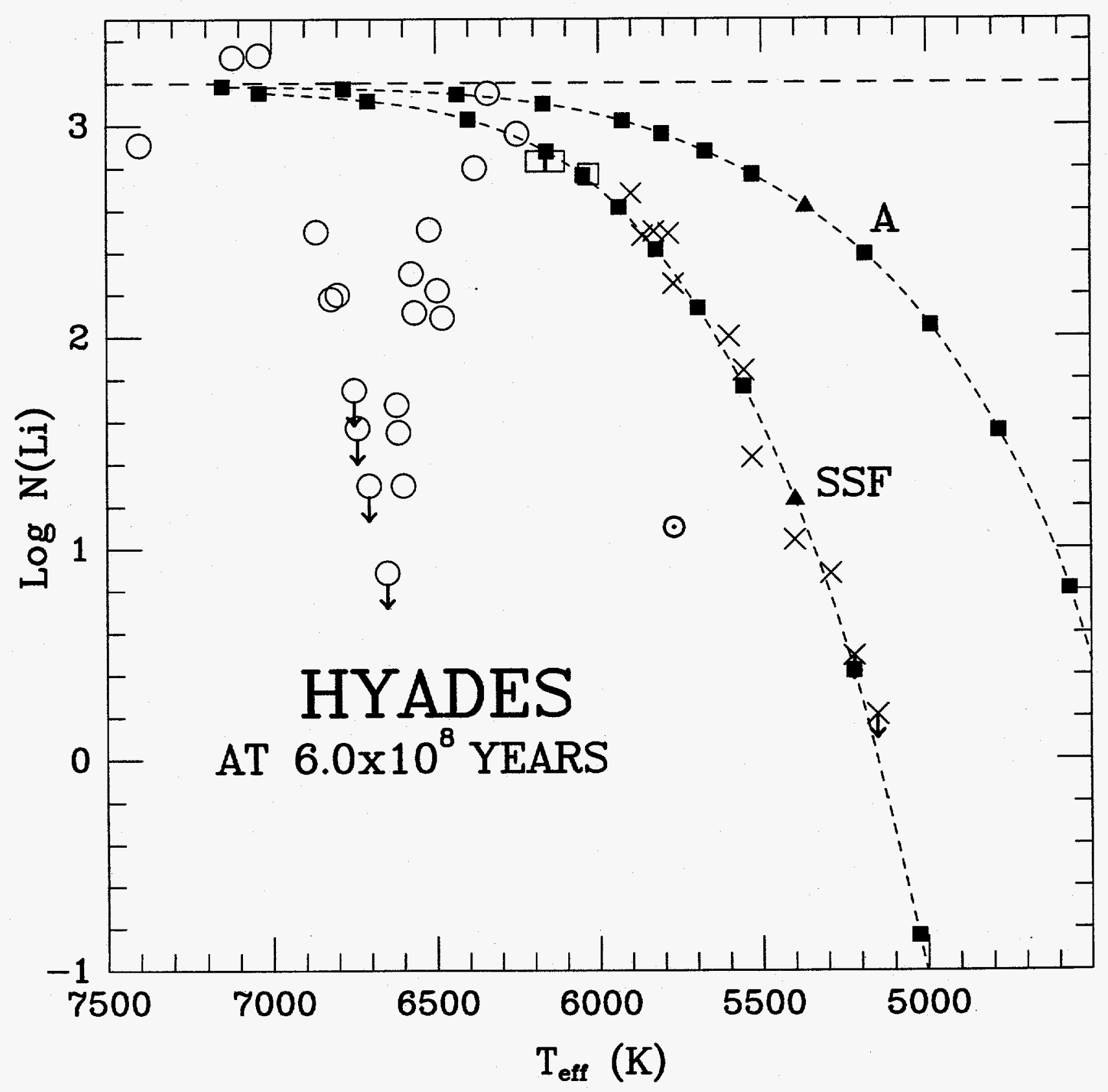


Fig 2.

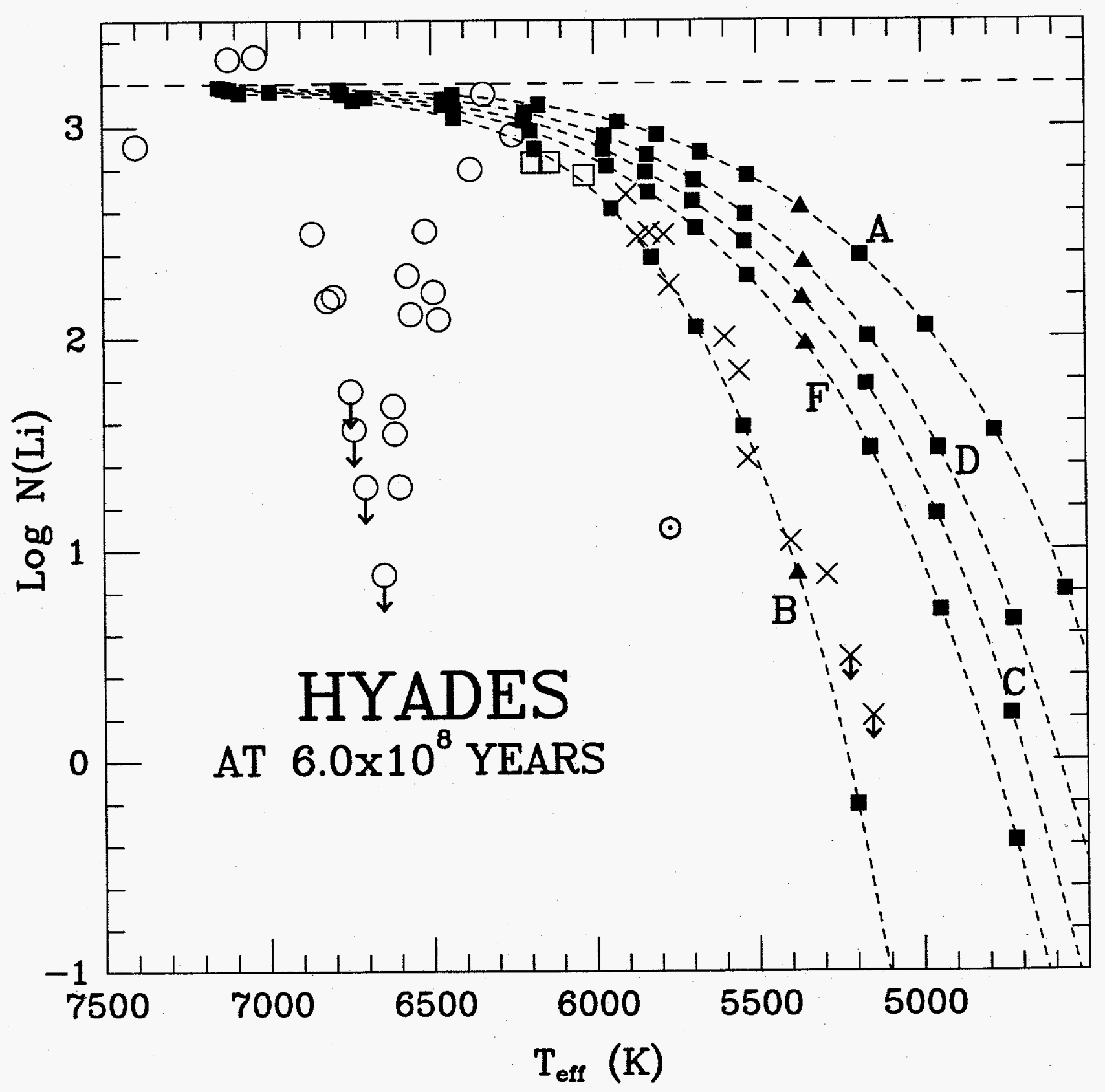


Fig 3

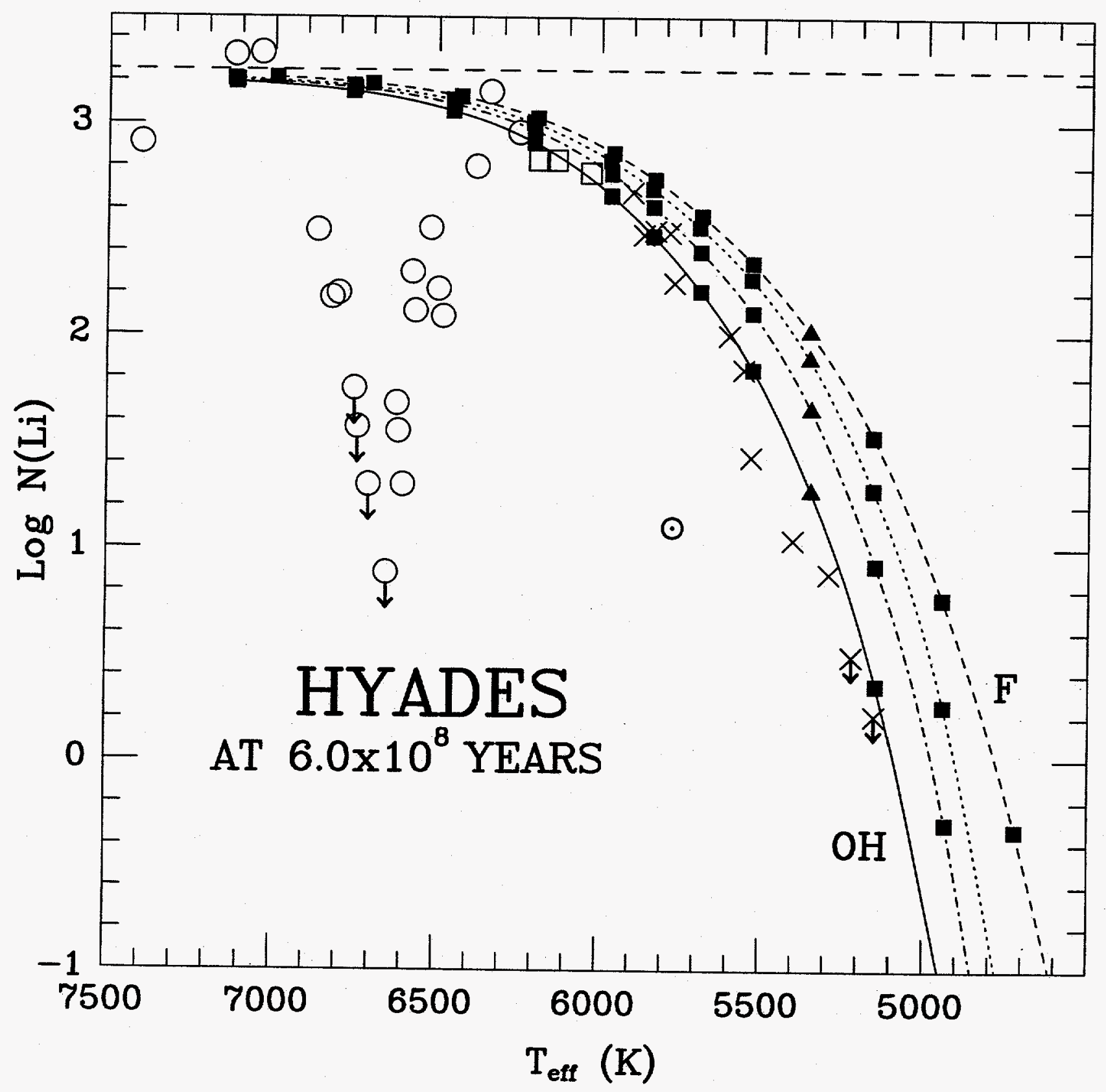

